\title{
Two-dimensional textural edge detection using $\omega$-Gaussian filter
}

\author{
Jinwoo Park, Ting-Chung Poon, and Guy Indebetouw
}

\begin{abstract}
A textural edge detection frequency domain filter is proposed to locate the boundary between two textural domains modulated by different spatial frequencies. This filter is derived from a zero-order prolate spheroidal function which can be approximated by a $\omega$-Gaussian shape. The extension to a 2-D rotational symmetric filter capable of locating textural edges independently of the relative orientation of the textural frequencies is demonstrated. Results show that the proposed filter is more effective at concentrating the output energy around the edge than the filter proposed by Shanmugan et al., which was derived with an additional constraint of evenness in the design of the filter function.
\end{abstract}

\section{Introduction}

Edge extraction is an important operation in a variety of image analysis and interpretation applications. Indeed, the most meaningful information is to a large extent contained in the edges and boundaries between areas of different textures in an image. Segmentation, or the extraction of these boundaries, can be done with local operators or frequency domain filters. ${ }^{1}$ The latter have the advantage of possible implementation via a fast Fourier transform or with fast parallel optical hardware.

Many types of filter have been used to extract the tonal edges or boundaries between two different gray levels in an image. The problem of extracting boundaries between two different textures is much more difficult but practically important. Since texture can usually be characterized by a few statistical parameters, ${ }^{2}$ local operator techniques capable of extracting such local parameters and finding boundaries have been very successful so far. ${ }^{3}$ However, it appears that use of the frequency domain type of filter useful for this task is much more restricted. Nevertheless, an interesting filter capable of extracting the boundary between domains modulated by different spatial frequencies has recently been proposed and demonstrated in 1-D simulations. ${ }^{4}$ This filter is an extrapolation of an optimum tonal edge extraction filter previously described by Shanmugan et al. ${ }^{5}$ An approximate form

The authors are with Virginia Polytechnic Institute and State University, Blacksburg, Virginia 24061; G. Indebetouw is in the Physics Department, and the other authors are in the Department of Electrical Engineering.

Received 17 November 1985.

0003-6935/86/142357-08\$02.00/0.

(C) 1986 Optical Society of America. of this filter, which is similar to the difference in two Gaussians (DOG) ${ }^{6}$ and the Laplacian of a Gaussian 7 filter, was found to perform particularly well in extracting the blurred or noisy edges. The optimum performance of the filter was demonstrated under the constraint of even symmetry, which was necessary to extend operation of the filter to a 2-D case. This constraint, however, is not necessary when considering the extension to a 2-D textural edge detection filter with rotational symmetry in the frequency domain. Without the requirement of even symmetry, the optimum filter is an odd function which can be approximated by an $\omega$-Gaussian shape. This point is discussed in the following sections, where we reassess the optimum conditions for performance of the filter. We then compare the performances of the odd-type ( $\omega$ Gaussian) filter with the even-type ( $\omega^{2}$-Gaussian) filter in some examples of textural edge detection.

\section{Optimal Filter}

\section{A. Tonal Edge Extraction}

It was shown by Shanmugan et al. ${ }^{5}$ that the optimal frequency domain filter $H_{\mathrm{sh}}(\omega)$ for tonal edge extraction is, under the constraints of band limitation and evenness of the function,

$$
H_{\mathrm{sh}}(\omega)= \begin{cases}\omega \psi_{1}(C, \omega I / 2 \Omega) & |\omega|<\Omega, \\ 0 & \text { otherwise, }\end{cases}
$$

where $\psi_{1}$ is the first-order prolate spheroidal wave function with the parameter relationship, $C=\Omega \cdot I / 2$. This filter, with a bandwidth $2 \Omega$, maximizes the filter output energy within a resolution interval $I$ around the edge. The confined energy is proportional to the eigenvalue $\lambda_{1}$, where $\lambda_{1}$ satisfies the relationship $P_{I}\left\{\psi_{i}\right\}=$ $\lambda_{i} \psi_{i}$ and where the operator $P_{I}\{\}$ describes a truncation to an interval $I$ and a convolution with $\sin \Omega x /(\pi x) .{ }^{8}$

The requirement of an even transfer function for the 
tonal edge filter is necessary only if this filter has to be extrapolated to be circular symmetric in two dimensions by mapping the linear spatial frequency into a radial frequency. The textural edge filter, however, is constructed by shifting the tonal edge transfer function to an offset characteristic textural frequency. Thus its transfer function can have even symmetry even if the tonal edge filter from which it is constructed is odd.

Without the constraint of evenness, the optimum edge detection filter is an odd-type filter based on the zero-order prolate spheroidal wave function having the form

$$
H_{\mathrm{op}}(\omega)= \begin{cases}\omega \psi_{0}(C, \omega I / 2 \Omega) & |\omega|<\Omega, \\ 0 & \text { otherwise, }\end{cases}
$$

where $\psi_{0}$ is the zero-order prolate spheroidal wave function. The output energy within a resolution interval $I$ about the edge is now proportional to $\lambda_{0}$. Since the eigenvalues $\lambda_{i}$ decrease rapidly with the index $i$, especially for small values of the parameter $C,{ }^{8}$ we can expect a sizeable gain in energy confinement within the interval $I$ with the odd filter transfer function given in Eq. (2).

For computational convenience, the closed form approximations of the prolate wave functions are used instead of the exact forms. Equations (1) and (2) become, respectively,

$$
H_{e}(\omega)=C_{1}(\omega / K)^{2} \cdot \exp \left[-(\omega / K)^{2}\right] \quad|\omega|<\Omega,
$$

where $H_{e}(\omega)$ is an approximation of Eq. (1) and represents the even-type filter or the $\omega^{2}$-Gaussian filter function, and

$$
H_{0}(\omega)=C_{2}(\omega / K) \cdot \exp \left[-(\omega / K)^{2}\right] \quad|\omega|<\Omega,
$$

where $H_{0}(\omega)$ is approximated from Eq. (2) representing the odd-type filter or the $\omega$-Gaussian filter function. In Eqs. (3) and (4), $C_{1}$ and $C_{2}$ are constants, and $K$ is filter parameter given by

$$
K=\Omega \sqrt{2 / C} .
$$

The parameter $K$ is a measure of the effective bandwidth of the filter. Note that the maximum of $H_{e}(\omega)$ occurs at $\omega=K$ and the maximum of $H_{0}(\omega)$ at $\omega=K / \sqrt{2}$. Therefore, the function parameter $K$ plays a key role in determining the characteristics of the filter.

\section{B. Textural Edge Extraction}

An idealized model of a textural edge in an image is the boundary between two domains, where each domain is modulated by a different spatial frequency. An appropriate textural edge detection filter should locate some observable marks along the textural edge in the output image plane.

The ideal 1-D textural image can be modeled with the following expression:

$$
f(x)=U(-x) \cos \left(\omega_{1} x\right)+U(x) \cos \left(\omega_{2} x\right)
$$

where $U(x)$ is a unit step function, and each sinusoid characterizes a different texture. The textural edge detection filter for such an input image must find the edge around $x=0$. The required filter $H(\omega)$ can be constructed from a combination of frequency shifted tonal edge filters. Since Eq. (6) is equivalent to a sum of the two modulated tonal areas, this can be done by centering (in the frequency domain) a tonal edge filter $H_{t}(\omega)$ at $\omega_{1}$ and another at $\omega_{2}$. It is clear that the symmetry of $H(\omega)$ and that of $H_{t}(\omega)$ are not related in general. The general transfer function of the textural edge detection filter has the form

$$
\begin{aligned}
H(\omega)= & H_{t}(\omega) *\left[\delta\left(\omega-\omega_{1}\right)+\delta\left(\omega-\omega_{2}\right)\right] \\
& +H_{t}(-\omega) *\left[\delta\left(\omega+\omega_{1}\right)+\delta\left(\omega+\omega_{2}\right)\right],
\end{aligned}
$$

where $H_{t}(\omega)$ can be either $H_{e}(\omega)$ or $H_{0}(\omega)$, and where * denotes a convolution operation and $\delta$ a Dirac delta function.

This filter is required not to pass any energy at zero frequency, corresponding to the dc input value, and at the textural frequencies $\omega_{1}$ and $\omega_{2}$. Hence the bandwidth $\Omega$ of $H_{t}(\omega)$ should be subject to the constraint of

$$
|\Omega|<\min \left[\omega_{1}, \omega_{2},\left|\omega_{1}-\omega_{2}\right| / 2\right] .
$$

The last term in the bracket of Eq. (8) prevents the terms in the filter from overlapping each other at middle points between $\omega_{1}$ and $\omega_{2}$. Such a constraint can be easily satisfied by controlling the parameter $K$ in Eqs. (3) and (4). However, the results of the next section show that, since Eq. (8) clearly affects the effective bandwidth of the filter, the best value for the parameter $K$ will generally be found through a tradeoff between the resolution and SNR.

The output spectrum of the linear filter can be written as

$$
G(\omega)=F(\omega) \cdot H(\omega),
$$

where $F(\omega)$ is the Fourier transform of the input. The corresponding $F(\omega)$ to Eq. (6) is

$$
\begin{aligned}
F(\omega)= & {\left[\pi \delta(\omega)+\frac{1}{j \omega}\right] *\left[\pi \delta\left(\omega-\omega_{1}\right)+\pi \delta\left(\omega+\omega_{1}\right)\right] } \\
& +\left[\pi \delta(\omega)-\frac{1}{j \omega}\right] *\left[\pi \delta\left(\omega-\omega_{2}\right)+\pi \delta\left(\omega+\omega_{2}\right)\right],
\end{aligned}
$$

where $j$ is $\sqrt{-1}$.

To derive a simple analytical expression for the output signal, it is assumed that $H_{t}\left(\omega-\omega_{i}\right) /\left(\omega-\omega_{j}\right)=0$ for $i \neq j$, i.e., $H_{t}(\omega)$ in Eq. (7) is bandlimited according to Eq. (8) so that the filter centered at $\omega_{1}$ overlaps negligibly with the part of the filter centered at $\omega_{2}$. Then the output spectrum of Eq. (9) becomes

$$
\begin{aligned}
G(\omega)= & \frac{\pi}{j}\left[H_{t}(\omega) / \omega\right] *\left[\delta\left(\omega-\omega_{1}\right)-\delta\left(\omega-\omega_{2}\right)\right] \\
& +\frac{\pi}{j}\left[H_{t}(-\omega) / \omega\right] *\left[\delta\left(\omega+\omega_{1}\right)-\delta\left(\omega+\omega_{2}\right)\right] .
\end{aligned}
$$

After taking the inverse Fourier transform of Eq. (11), the output in the space domain is found to be

$$
g(x)=\operatorname{Odd}\left\{g_{t}(x) \cdot\left[\exp \left(j \omega_{1} x\right)-\exp \left(j \omega_{2} x\right)\right]\right\},
$$

where Odd means the odd part, and $g_{t}(x)$ is the inverse Fourier transform of $H_{t}(\omega) / \omega$. 
With the $\omega^{2}$-Gaussian function of Eq. (3), i.e., $H_{t}(\omega)$ $=H_{e}(\omega)$, the output of Eq. (12) becomes

$$
g_{e}(x)=\frac{K x}{2} \exp \left(-\frac{K^{2} x^{2}}{4}\right)\left[\cos \left(\omega_{1} x\right)-\cos \left(\omega_{2} x\right)\right] .
$$

Similarly, the $\omega$-Gaussian filter function of Eq. (4), i.e., if $H_{t}(\omega)=H_{0}(\omega)$, leads to the output

$$
g_{0}(x)=\exp \left(-\frac{K^{2} x^{2}}{4}\right)\left[\sin \left(\omega_{1} x\right)-\sin \left(\omega_{2} x\right)\right] .
$$

Some inessential constants are omitted for simplicity in Eqs. (13) and (14).

\section{Comparison of Odd and Even Filters}

An analytical way of comparing the textural edge detection filters can be based on evaluating the ratio $\alpha$ of the confined output energy in an interval $\Delta x$ around the edge to the total output energy:

$$
\alpha(\Delta x, K)=\left.\int_{-\Delta x / 2}^{\Delta x / 2}|g(x)|^{2} d x\left|\int_{-\infty}^{\infty}\right| g(x)\right|^{2} d x .
$$

In the case that $\omega_{1}, \omega_{2} \gg K$ so that the integrals of $\sin ^{2}$ (.) and $\cos ^{2}(\cdot)$ average to $1 / 2$ under the slowly varying Gaussian envelope, Eq. (15) becomes

$$
\alpha_{e}(N)=\operatorname{erf}(N)-N \frac{d}{d N}[\operatorname{erf}(N)]
$$

if $g(x)=g_{e}(x)$, and

$$
\alpha_{0}(N)=\operatorname{erf}(N)
$$

if $g(x)=g_{0}(x)$. In these expressions, $N=K \cdot \Delta x / 2 \sqrt{2}$ is a measure of the number of resolution elements in the interval $\Delta x$, and

$$
\operatorname{erf}(N)=\frac{2}{\sqrt{\pi}} \int_{0}^{N} \exp \left(-t^{2}\right) d t
$$

is the error function. A plot of the energy confinement ratio $\alpha(N)$ is shown in Fig. 1. Note that the $\omega$-Gaussian filter clearly yields a much better energy confine-

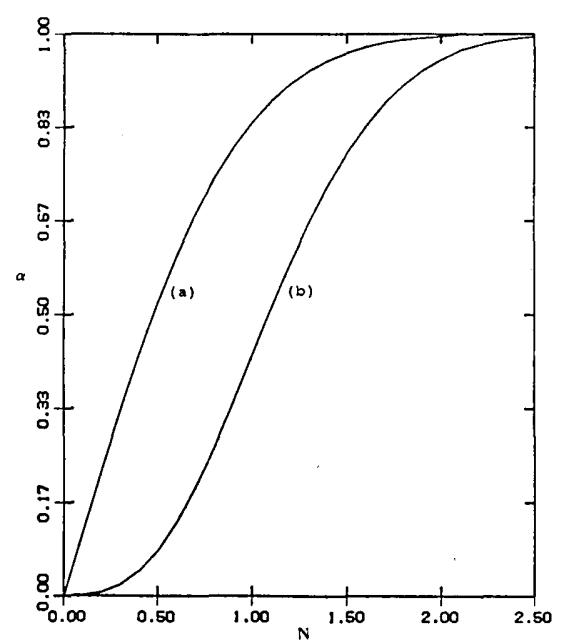

Fig. 1. Comparison of the relative energy confined in an interval $\Delta x$ around the edge image in terms of the number of resolution elements $N$ in $\Delta x$ : (a) $\omega$-Gaussian filter; (b) $\omega^{2}$-Gaussian filter. ment than the $\omega^{2}$-Gaussian filter, especially for small values of $N$, i.e., for a narrow interval $\Delta x$.

Some 1-D results are presented here in Figs. 2-6. The input image described by Eq. (6) is shown in Fig. 2 , where the textural frequencies, $\omega_{1}$ and $\omega_{2}$, are $\pi / 2$ and $\pi / 6$, respectively. An example of the $\omega^{2}$-Gaussian filter for the textural edge detection with $K=\pi / 18$, complying with the bandlimit constraint of Eq. (8), is shown in Fig. 3(a). The resulting output normalized to yield a peak response of unity is shown in Fig. 3(b). For comparison, a $\omega$-Gaussian filter, shown in Fig. 4(a), was designed with the same filter parameter $K=\pi / 18$. The resulting output is shown in Fig. $4(\mathrm{~b})$, where the

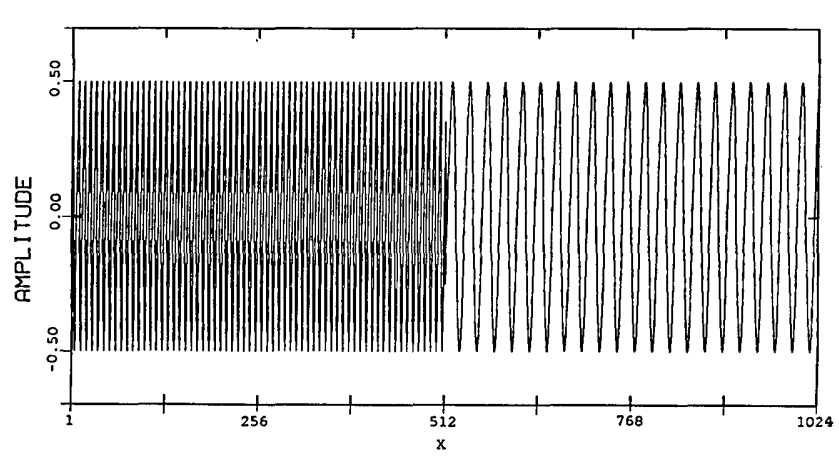

Fig. 2. One-dimensional input image consisting of a textural edge characterized by two frequencies, $\omega_{1}=\pi / 2$ and $\omega_{2}=\pi / 6$.

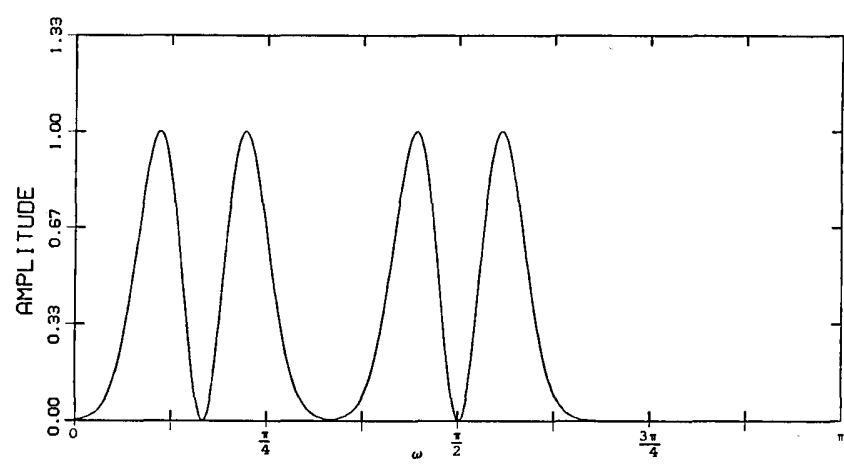

(a)

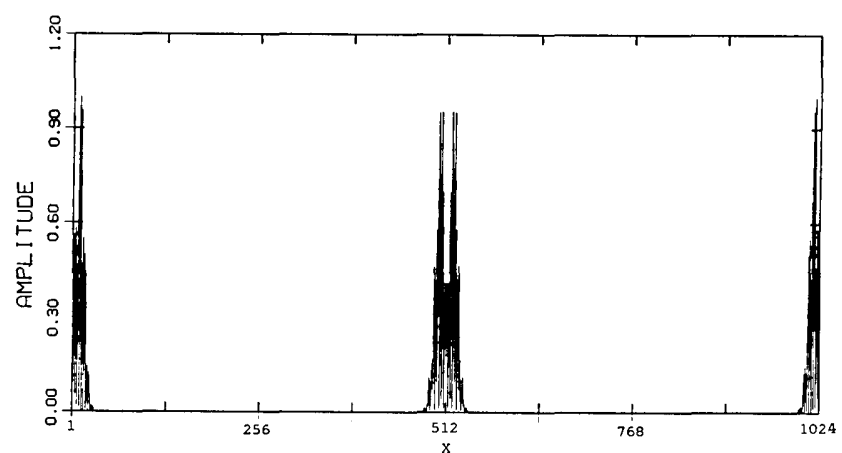

(b)

Fig. 3. $\omega^{2}$-Gaussian filter function: (a) 1-D filter transfer function with $K=\pi / 18$ designed to extract the textural edge in Fig. 2; (b) output of the textural edge detection. 
magnitude of the output is normalized to the maximum value of the output of the $\omega^{2}$-Gaussian filter of Fig. 3(b). As expected from Eq. (13), the envelope of the $\omega^{2}$-Gaussian filter output is a Gaussian multiplied by $x$ resulting in two peaks modulated by the difference of the two cosine functions. In contrast, the $\omega$ Gaussian filter produces an output with only one peak described by a Gaussian envelope modulated by the difference of the two sine functions. In comparing the two filter outputs, it is apparent that the output energy of the $\omega$-Gaussian filter is more concentrated near the center of the edge. This results in a higher peak within a narrower interval and thus a higher resolution of the extracted edge. The $\omega$-Gaussian filter appears to have relatively higher background ripples on one side. This is due to the input spectrum leakage through the regions of the filter near $\omega_{1}$ and $\omega_{2}$, where the slope of the filter function is very high. In simulation with FFT, this phenomenon could be suppressed to some extent by increasing the number of samples.

The resolution of the filter can be enhanced by increasing the parameter $K$. This results in a larger bandwidth for the filter function but a sharper image of the detected edge. However, a larger bandwidth in the filter usually involves truncation of parts of the filters. Such truncations at the midfrequency between $\omega_{1}$ and $\omega_{2}$ as well as at $\omega=0$ and $\pi$ are necessary to avoid overlapping between the adjacent tonal filters deployed in a textural edge detection filter. Therefore, the gain of resolution is then achieved at the expense of a higher artifact noise due to discontinuities in the filter function, a loss of the filter's optimum performance due to truncation of some part of the optimum function, and a reduction of the SNR due to the wider bandwidth. Generally, a trade-off needs to be made between these various factors depending on which factor should be emphasized. This important aspect of the filter's performance is not being considered in this analysis.

With a filter parameter $K=\left|\omega_{1}-\omega_{2}\right| / 2=\pi / 6$, the two parts of the $\omega^{2}$-Gaussian filter connect smoothly at their maxima as shown in Fig. 5(a). The higher-resolution output is shown in Fig. 5(b). A similar gain of resolution can be achieved with the $\omega$-Gaussian filter. However, because of its odd symmetry, a severe discontinuity will occur at $\omega=\left|\omega_{1}-\omega_{2}\right| / 2$. To avoid this, the sign of one part of the filter can be inverted. [For example, the sign of the second term centered at $\omega_{2}$ in Eq. (7) is changed from plus to minus.] The transfer function with $K=\pi / 3 \sqrt{2}$, constructed in the way described to insure a smooth connection of the curve at the maximum at $\omega=\pi / 6$, is shown in Fig. 6(a). Figure $6(\mathrm{~b})$ is the output with enhanced resolution.

\section{Two-Dimensional Rotational-Symmetric Filter}

The textural edge extraction filter of Eq. (7) can be extended to two dimensions by converting $\omega$ to a radial spatial frequency leading to a rotational-symmetric filter. The resulting filter is rotationally invariant and can extract boundaries between 2-D textural image areas modulated by different spatial frequencies, inde-

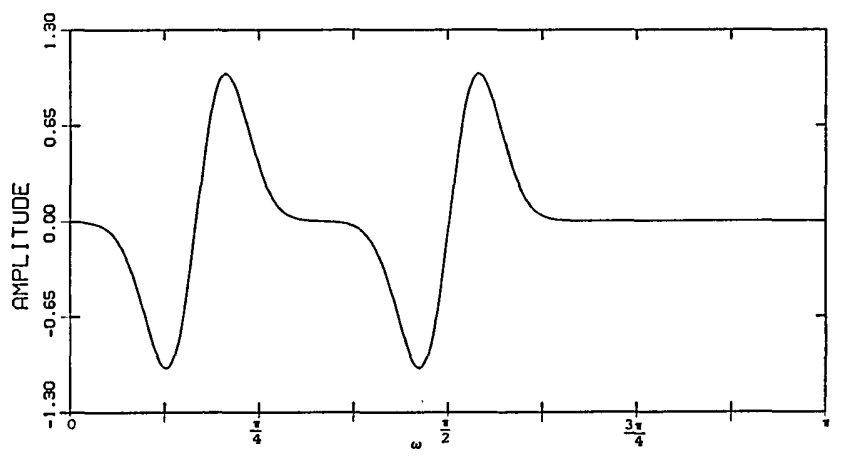

(a)

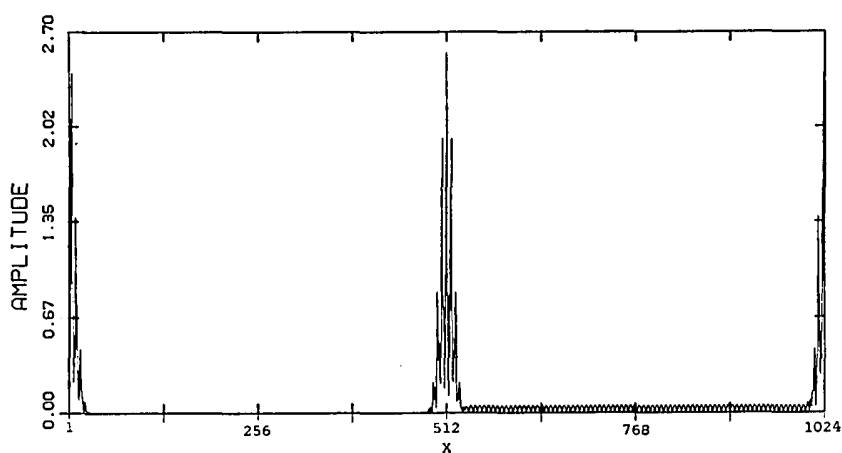

(b)

Fig. 4. $\omega$-Gaussian filter function: (a) 1-D filter transfer function with $K=\pi / 18$ to extract the textural edge in Fig. 2; (b) output of the textural edge detection.

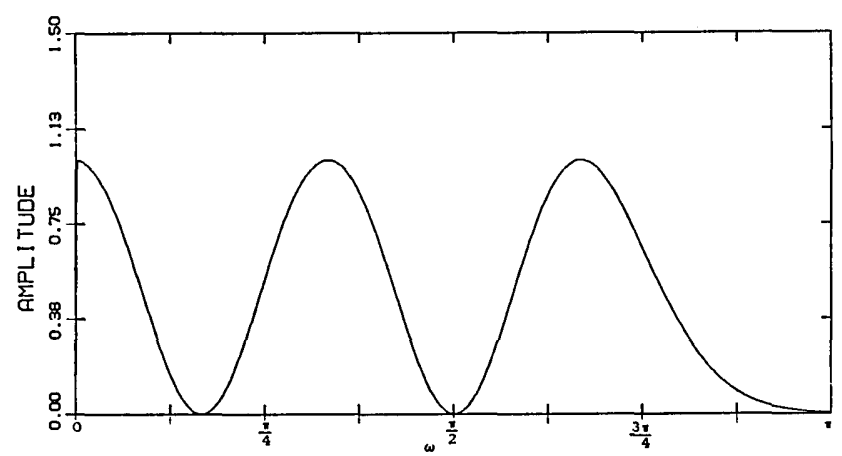

(a)

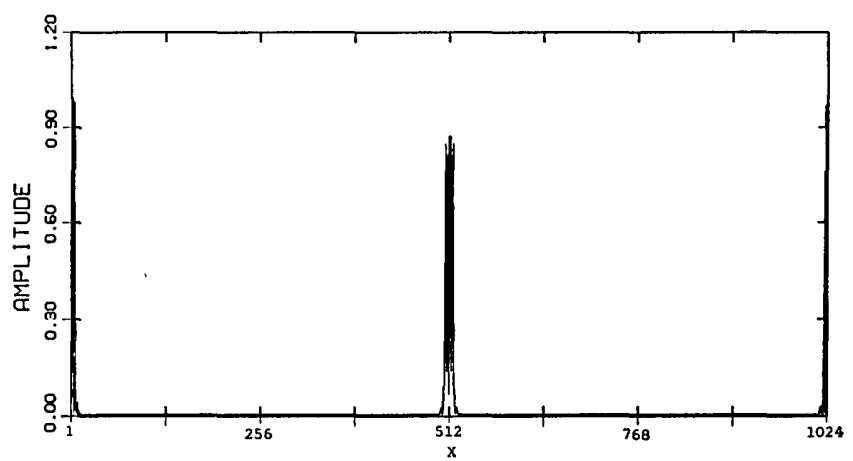

(b)

Fig. 5. $\omega^{2}$-Gaussian filter with a wider bandwidth: (a) $\omega^{2}$-Gaussian filter transfer function with $K=\pi / 6$; (b) filter response with higher resolution. 
pendently of the relative orientation of the textural frequencies. The 2-D expression corresponding to the 1-D Eq. (7) may be written as

$$
H_{\text {rot }}(\rho)=H_{t}\left(\rho-\omega_{1}\right)+H_{t}\left(\rho-\omega_{2}\right),
$$

where $\rho=\left(\omega_{x}^{2}+\omega_{y}^{2}\right)^{1 / 2}$. The basic idea in implementing the rotational-symmetric filter is as follows. If the orientation of the textural frequency in an image is changing while keeping the same textural characteristics (i.e., the same characteristic spatial frequencies), the spectrum of the textural image in the 2-D frequency domain moves on a circle of constant radius. Therefore, a rotational-symmetric filter must be invariant to the relative orientation of the textural frequencies. An example of a textural image used in the following experiments is shown in Fig. 7, with the two different textural frequencies of $\pi / 2$ and $\pi / 6$, orientated $45^{\circ}$ to each other.

The 1-D filter based on the zero-order prolate spheroidal wave function is an optimum filter leading to a maximum output energy density in an interval around the localized edge. Preliminary results show that the 2 -D rotational-symmetric filter based on this 1-D optimum transfer function performs well. It is further expected that it will be particularly useful in detection of the blurred or noisy edges. Most of the statements and discussions mentioned for the 1-D case, regarding the filter performance filter design procedures, still hold for the 2-D analysis.

An example of a 2-D $\omega^{2}$-Gaussian filter with $K=\pi /$ 18 is presented in Fig. 8(a). This filter is designed to detect the edges between the two textures in the image shown in Fig. 7 regardless of the relative orientations between these textures. Figures $8(\mathrm{~b})$, (d), and (f) show the outputs for the three test images having relative textural orientations of 0,45 , and $90^{\circ}$, respectively. The corresponding contour representations are shown in Figs. 8(c), (e), and (g). Note that the filter responses are independent of the textural orientation. The width of the detected edge is approximately the same for all the orientations, but the structure within the detected edge depends on the relative orientation.

The $\omega$-Gaussian filter with $K=\pi / 18$, shown in Fig. 9(a), was also applied to the test image of Fig. 7. The results for an orientation of $45^{\circ}$ are shown in Figs. 9(b) and (c). The $\omega$-Gaussian filter clearly generates an output with a higher energy concentration along the detected edge, i.e., a higher resolution. If a filter function with a wider bandwidth is desired, the same truncation procedures used in the 1-D case can be applied. A much higher resolution can then be achieved, as shown in Figs. 10 and 11. Figures 10(a) and (b) show the output of the $\omega^{2}$-Gaussian filter with $K=\pi / 6$, while Figs. 11(a) and (b) is the output of the corresponding $\omega$-Gaussian filter with $K=\pi / 3 \sqrt{2}$.

\section{Conclusions}

We have shown that the filter which maximizes the output energy near the boundary between two image areas modulated by two different spatial frequencies is proportional to a bandlimited zero-order prolate sphe-

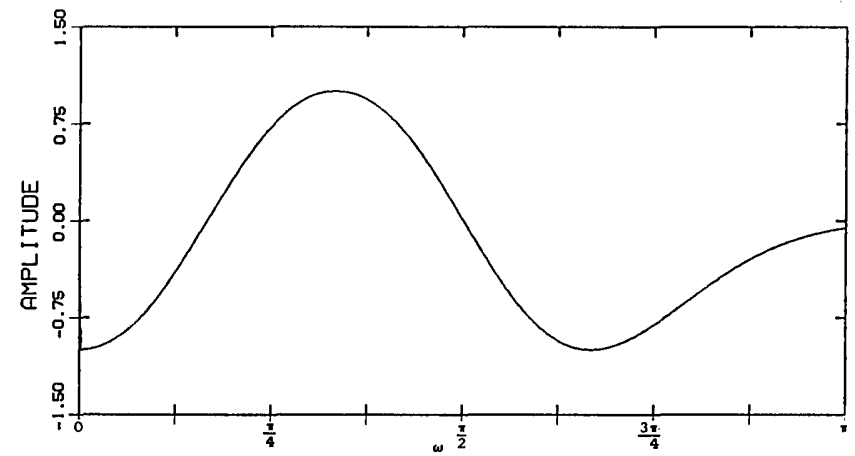

(a)

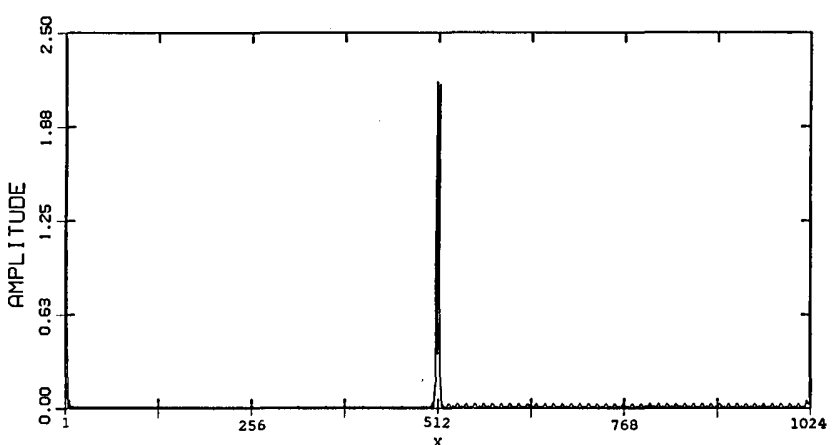

(b)

Fig. 6. $\omega$-Gaussian filter with a wider bandwidth: (a) $\omega$-Gaussian filter transfer function with $K=\pi / 3 \sqrt{2}$; (b) the filter response with higher resolution.

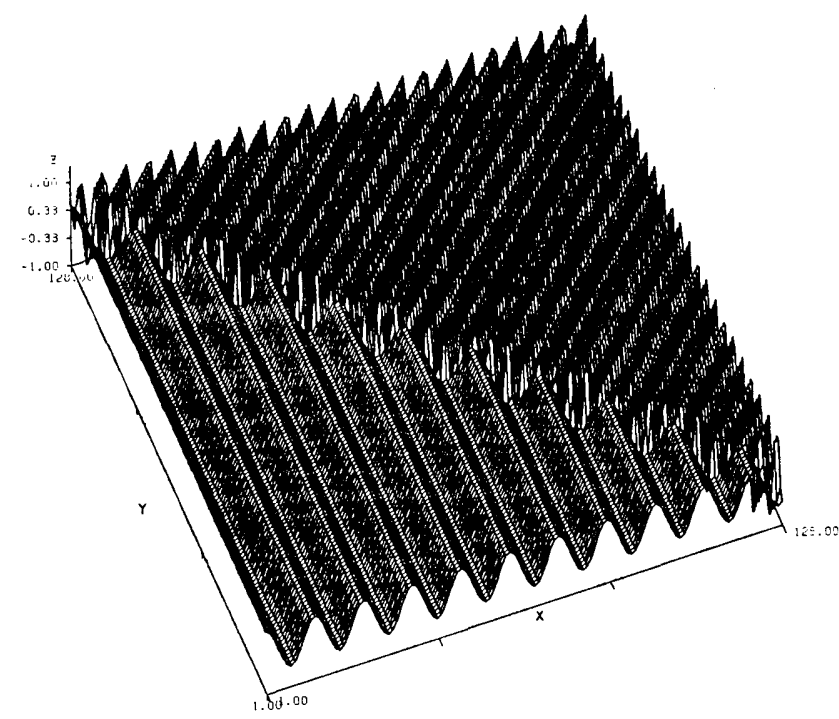

Fig. 7. Two-dimensional textural input image consisting of an edge between two texture areas modulated by the frequencies $\pi / 2$ and $\pi /$ 6 , respectively, with relative orientation of $45^{\circ}$. 


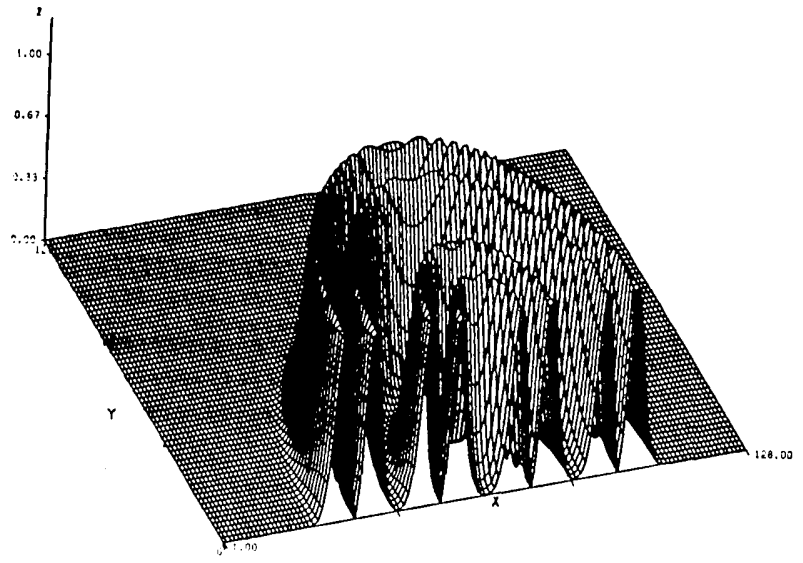

(a)

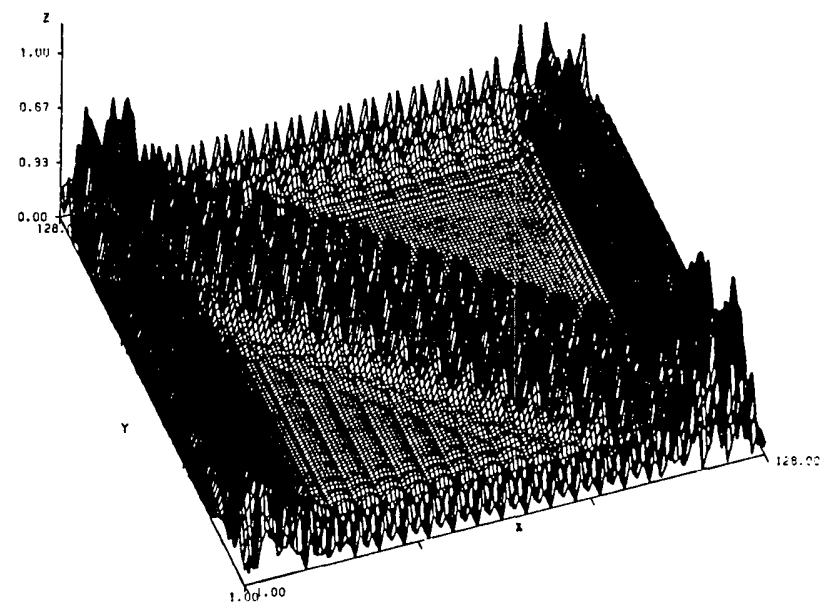

(b)

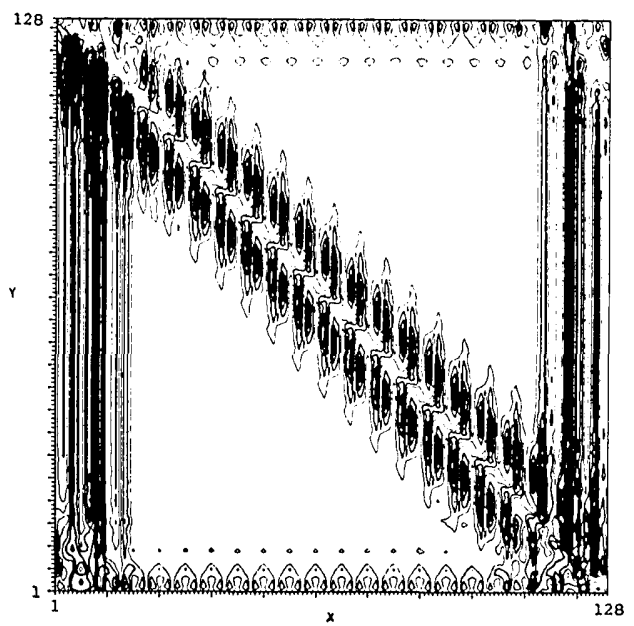

(c)

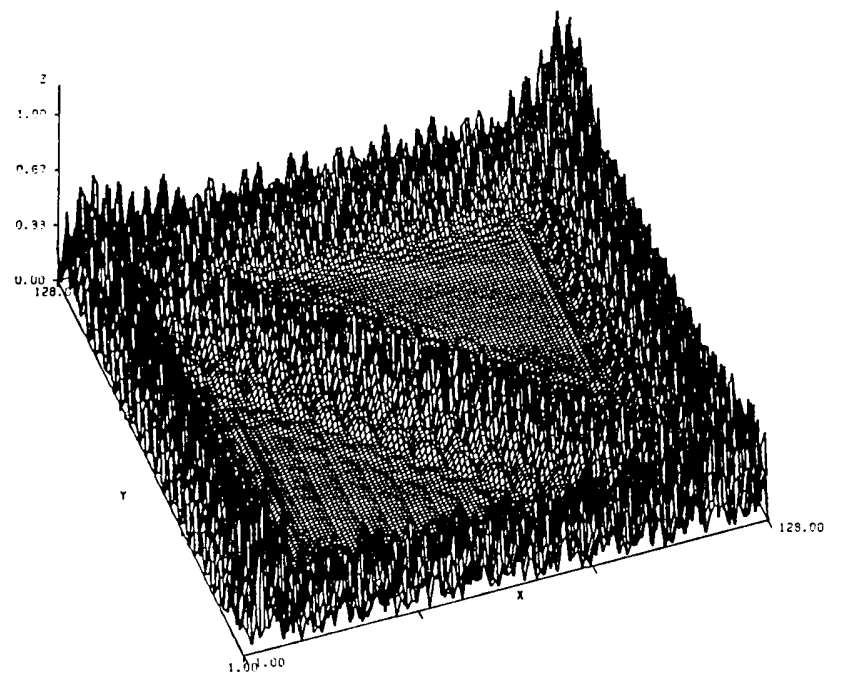

(d)

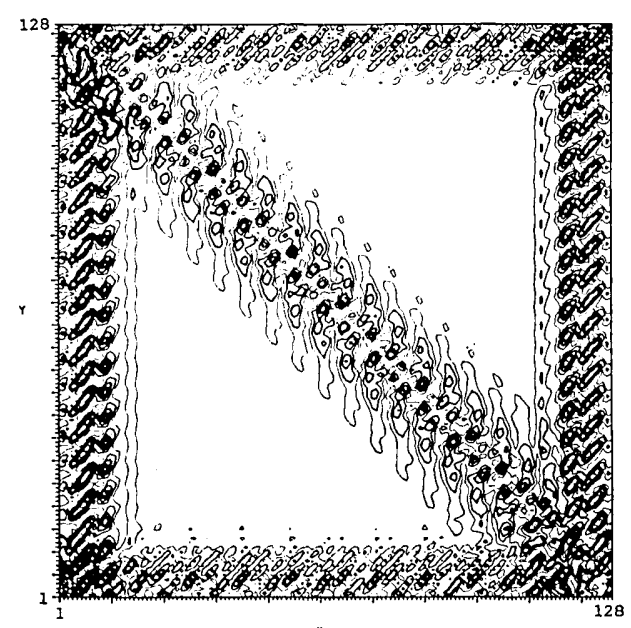

(e)

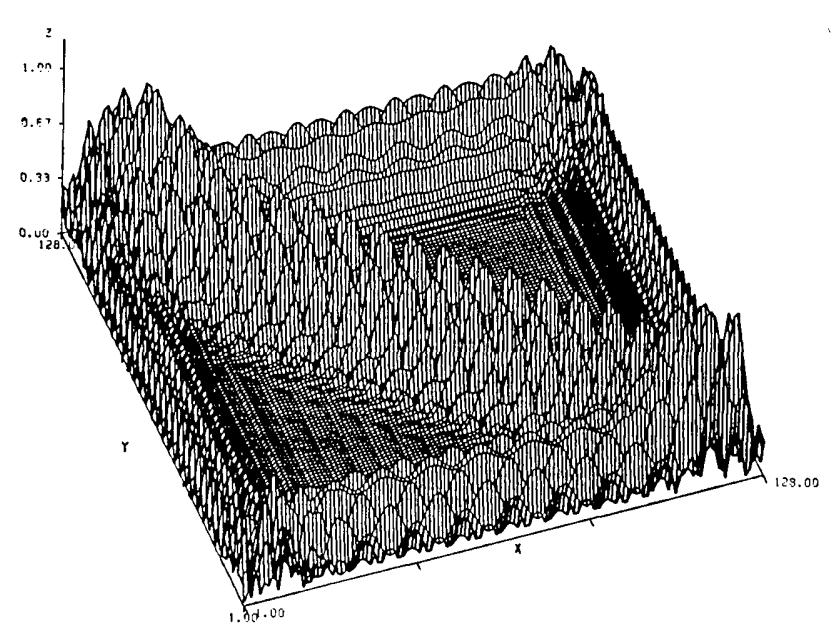

(f)

Fig. 8. Cross section of a 2-D rotational-symmetric $\omega^{2}$-Gaussian filter and its response: (a) $\omega^{2}$-Gaussian filter transfer function with $K=\pi / 18$ designed to detect the edge between the two textures of Fig. 7 independently of their relative orientation; (b), (d), and (f) filter responses to three input images with orientation angles of 0,45 , and $90^{\circ}$, respectively; (c), (e), and (g) contour representations of the outputs (b), (d), and (f), respectively. 


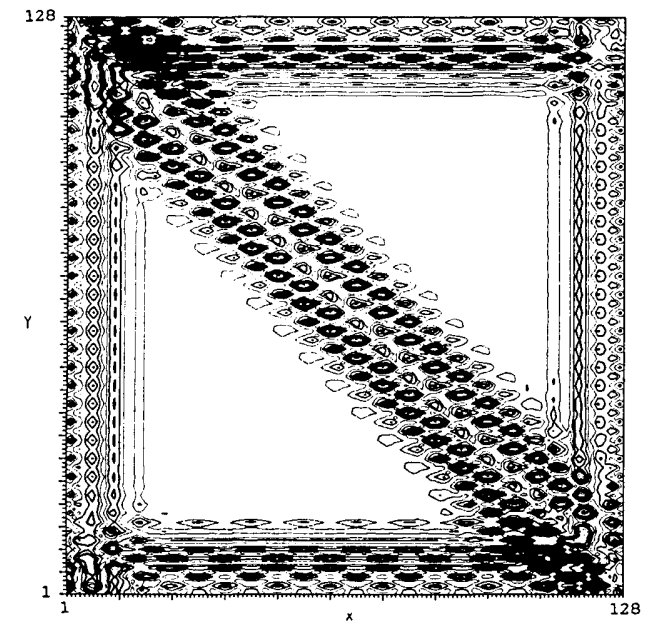

(g)

Fig. 8 continued. See caption on page 2362 .

roidal wave function centered at both frequencies. The energy confinement at the boundary produced by this filter is better than that obtained with the filter function proposed by Shanmugan et al., which is optimum under the additional constraint of evenness. Both filters can be extended to 2-D rotational-symmetric filters by appropriately bandlimiting their transfer functions and changing the linear spatial frequency into a radial spatial frequency.

Further analysis is clearly needed to determine the usefulness of such filters for texture segmentation. Since textures which can be characterized by a unique spatial frequency may not be very common in nature, the deterioration of the filters performances when applied to less deterministic types of texture needs to be studied.

Nevertheless, one of the most significant advantages of global frequency domain filters is that they can be easily implemented in a variety of optical systems including coherent parallel filtering setups ${ }^{9}$ and incoherent parallel or scanning systems using two-pupil interaction schemes. ${ }^{10}$

The partial financial support of the National Science Foundation (grant ESC 8400635 for J. Park and T.-C. Poon and grant ECS 8116337 for G. Indebetouw) for the work on which this report is based is gratefully acknowledged.

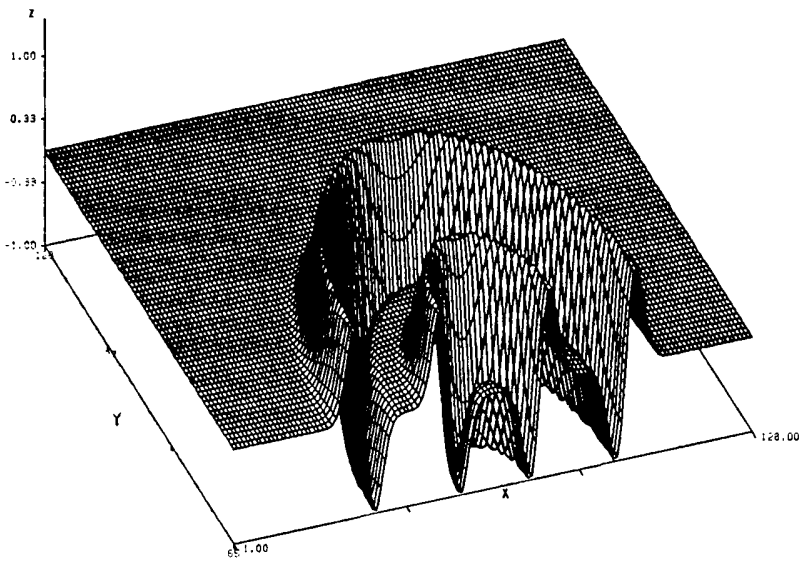

(a)

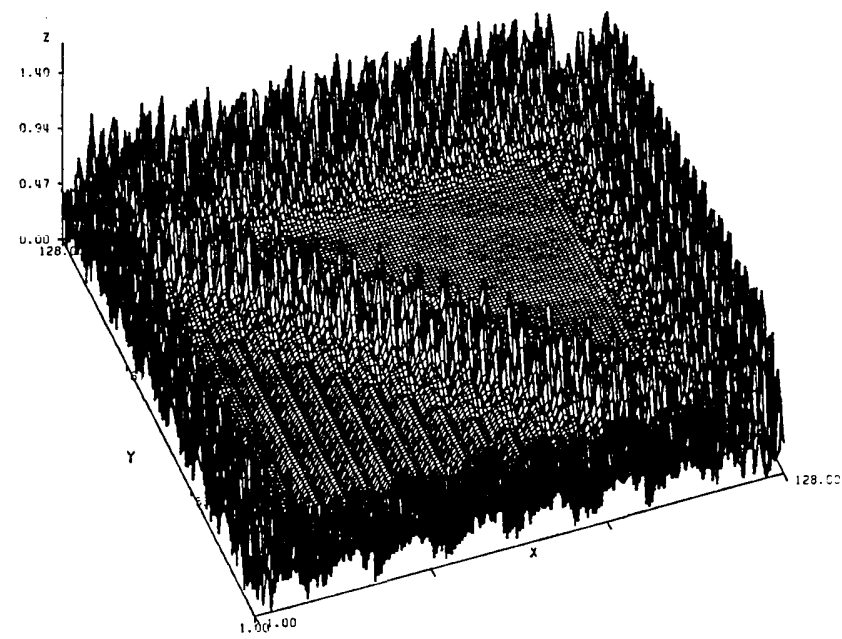

(b)

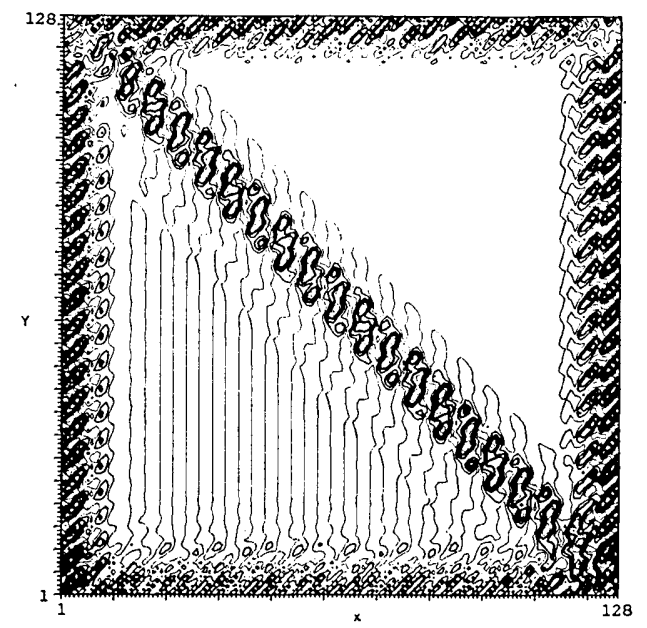

(c)

Fig. 9. Cross section of a 2-D rotational-symmetric $\omega$-Gaussian filter function and its response: (a) $\omega$-Gaussian filter transfer function with $K=\pi / 18$; (b) filter response to the textural image in Fig. 7;

(c) contour representation of the filter response. 


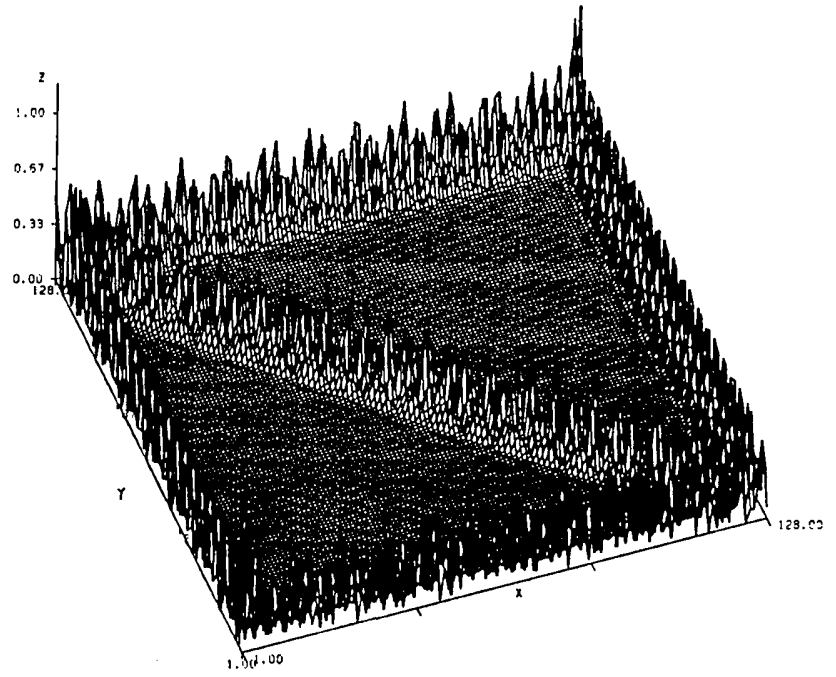

(a)

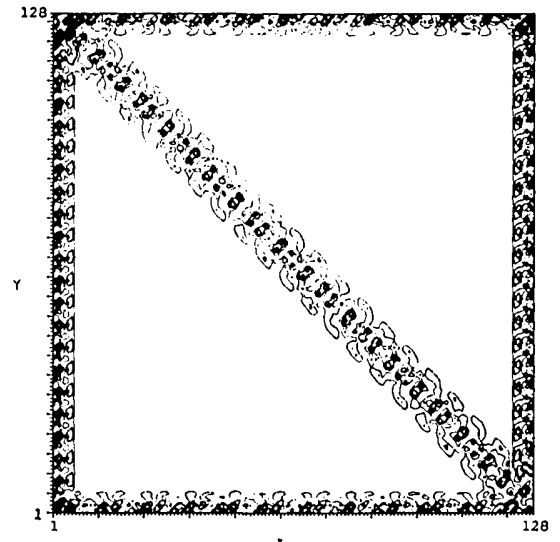

(b)

Fig. 10. Edge detection by a wider bandwidth $\omega^{2}$-Gaussian filter with $K=\pi / 6$ : (a) filter response to the textural image in Fig. 7; (b) contour representation of the filter response.

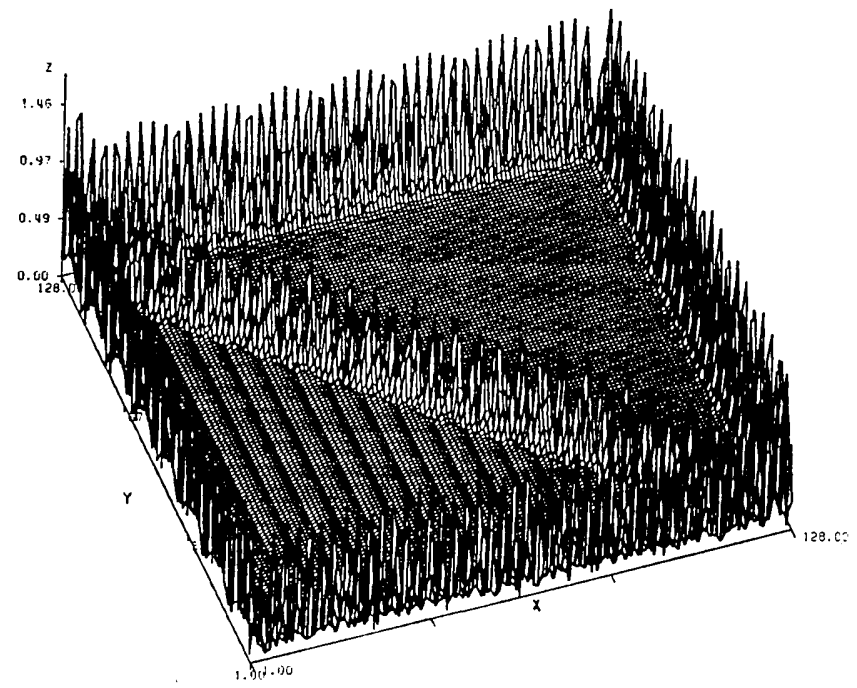

(a)

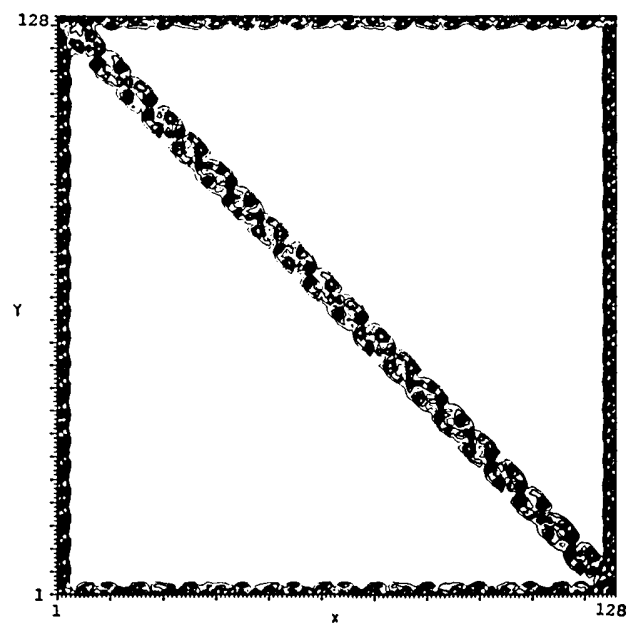

(b)

Fig. 11. Edge detection by a wider bandwidth $\omega$-Gaussian filter with $K=\pi / 3 \sqrt{2}$ : (a) filter response to the textural image in Fig. 7; (b) contour representation of the filter response.

tures," IEEE Trans. Pattern Anal. Machine Intell. PAMI-1, 37 (1979).

6. I. Overington, "Toward a Complete Model of Photopic Visual Threshold Performance," Opt. Eng. 21, 2 (1982).

7. D. Marr and E. C. Hildreth, "Theory of Edge Detection," Proc. R. Soc. London Ser. B 207, 187 (1980).

8. D. Slepian and H. O. Pollak, "Prolate Spheroidal Wave Functions, Fourier Analysis and Uncertainty-I," Bell Syst. Tech. J. 40, 43 (1961).

9. J. W. Goodman, Introduction to Fourier Optics (McGraw-Hill, New York, 1968).

10. A. W. Lohmann and W. T. Rhodes, "Two-Pupil Synthesis of Optical Transfer Functions," Appl. Opt. 17, 1141 (1978). 